\title{
Conceptual Agent-Based Model for Optimal Power Utilization in Wireless Sensor Networks
}

\author{
Olumide S. Adewale ${ }^{*}$ Ojonukpe S. Egwuche*, Samuel A. Oluwadare*
}

\begin{abstract}
Wireless Sensor Networks (WSNs) are densely and largely deployed in a variety of environments to sense real-world events. Many approaches have been proposed for collecting sensory data in any wireless sensor networks in a flexible, reliable, and efficient manner. In this study, we design an efficient mobile agent-based model for data gathering in wireless sensor networks to achieve energy and timely collection of sensory information in the target scene. The current focus in wireless sensor networks is placed on energy optimization during data gathering, processing and transmission. Mobile Agents (MAs) as a piece of a program have attracted growing research interest that travels the network from node to node to compute the local data to get useful information globally. Mobile agent's features such as autonomy, social ability, learning, and more significantly, mobility makes it a chosen technology for information processing in wireless sensor networks and other resource constrained computing environments. The nodes of the sensors are modelled and represented by the states of a Markov chain. The nodes are further grouped into clusters to save the energy of the nodes farther away from the base station.
\end{abstract}

Keywords: Mobile Agents, Sensor Nodes, Wireless Sensor Networks, Clustering.

\section{Introduction}

Recent advances in wireless communications and electronics have led to the development of Wireless Sensor Networks (WSNs). Wireless network refers to any type of computer network that is not connected by cables of any kind. A wireless sensor network (WSN) consists of spatially distributed autonomous sensor nodes and a communication system that allows automatic data collection, processing and sharing based on the collaborative effort of a large number of sensor nodes. Sensor networks are used for collecting, storing and sharing the sensed data and to cooperatively pass their data through the network to a main location (Zhu, et al, 2012).

Lange and Oshima (1999) defined mobile agent as a program that assists people and acts on their behalf on a network. An agent possesses the following properties:

a. Reactive - senses changes in the environment and acts accordingly to those changes;

b. Autonomous - has control over its own actions;

c. Goal driven - is pro-active;

d. Temporally continuous - is continuously executing;

e. Communicative - able to communicate with other agents;

\footnotetext{
* Computer Science Department, Federal University of Technology Akure, P.M.B. 704, Akure, Ondo State, Nigeria

$\bowtie$ osadewale@futa.edu.ng, osegwuche@futa.edu.ng, saoluwadare@futa.edu.ng
} 
f. Mobile - can travel from one host to another;

g. Learning - adapts in accordance with previous experience

The applications of wireless sensor networks are increasing in wide areas of human endeavours. They are especially useful for human operations in unreachable fields and hazardous environments (Dong, 2014). Chen, Gonzalez, and Leung (2009) highlighted that a notable challenge in the design of wireless sensor networks in any capacity is minimizing power consumption towards maximizing the network lifetime. WSNs can produce a measurable response to changes in physical conditions and the primary goal of such networks is to perform distributed sensing tasks. These networks constitutes a new kind of ad hoc networks with a new set of characteristics and challenges

The remainder of the paper is arranged as follows. Section 2 shows the reviews of related works and how the observed limitations form the basis of our design. Section 3 presents the design and proposed energy optimization algorithm. Finally, we conclude our paper and mention directions for future work in Section 4.

\section{Literature Review}

Ahmed (2013) presented a dynamic wireless sensor network in which the sensor nodes and the base stations are mobile. Their study provides a load distribution real-time routing protocol for mobile wireless sensor networks. There is continuous selection of nodes with the highest remaining power. The continuous selection of such nodes spread out the traffic load to neighbours in the direction of the sink, and subsequently prolonging the network lifetime. Data aggregation is an effective way of reducing the number of redundant data in the system.

In Wireless Sensor Network, data fusion or data aggregation is the key technology to decreased power consumption and improved accuracy of data collection. In spite of the existence of models used for distributed systems for ordinary computing platforms that could solve the problems related to the distributed nature of WSN applications, they do not address the needs related to the constrained energy and processing resources presented by WSN nodes. Recognizing such situation, research is being developed towards adaptation of such models to the WSN reality. Zhu et al (2012) noted that Mobile agents are capable of migrating across the network carrying code and state. As they migrate, they perform application-specific tasks like taking sensor readings, performing in-network data aggregation, and coordinating with other agents to achieve a common goal. Network exploration requires that every region of the sensor network be visited at least once in a manner that is efficient in terms of energy consumption and latency.

Lin et al (2012) observed that most sensor nodes are unable to communicate with the sink node directly because of their limited communication capacity. Intermediate nodes deplete their energy faster when taking more tasks. The work assumes that all the sensor nodes generate data at the same rate and the size of each data packet is same. What now happens when the rate and the size are not the same? That becomes a research question to be answered by other authors.

Alipour and Faez (2011) observed that complexities on the design of a mobile agent routing algorithm are related to the precise selection of source nodes and their visiting sequence during mobile agent migration. The results of their simulations carried out in NS-2 shows that for networks with different number of nodes, the proposed method has less delay and energy consumption. The simulation parameters include terrain area, number of nodes, transmission 
range, MAC, and simulation time. But the study did not cover multi-cooperative mobile agent to achieve more precision and less delay.

Sutagundar and Manvi (2013) noted that using conventional methods of data gathering and processing in WSNs may lead to some of the limitations like excess energy consumption, redundant data transmission, increased latency, and bandwidth overheads. The critical issue in a WSN is the network lifetime which is mainly dependent on the energy of sensor nodes. The scheme made use of a set of mobile agents for collecting partial topology information connecting event and processing node by using location information. The cost of location technique was reduced by implementing GPS modules in selected few sensor nodes. Part of the limitations of the work is that all the nodes in the network (sensor nodes and sink node) are static and information could only be gathered from the partial topology of the network.

Imianvan (2008) was motivated by the wide applications of mobile agents in various field of human interest, such as e-commerce, distributed information retrieval, and proper network management that requires monitoring and controlling of network resources. The objectives of the study were to provide an intelligent system that can manage the use of bandwidth in a computer network environment, and to generate desirable statistics for policy formulation and decision making. The result obtained from the experimental study provides information for ranking workstations by bandwidth consumption, prioritization and sharing. The research was however limited in a fact that bandwidth is one of the many resources of the network that could be managed, the mobile agent is developed using visual basic programming language which is not well suited for mobile agent technology, and the mobile agent is launched from a server machine which imposes a limitation on the system.

Mini et al., (2003) proposed a prediction-based energy map for wireless sensor networks. If a node is able to predict the amount of energy it will spend, it can send this information directly to the monitoring node. The information about the amount of available energy in each part of the network can be useful to increase the lifetime of the network. However, the modes of operation specified in the implementation procedures cannot survive scalable and dynamic networks like wireless sensor networks.

Chen, Gonzalez and Leung, (2009) highlighted an energy efficient clustering algorithm for maximizing lifetime of wireless sensor networks. Their objective was to design an energy efficient clustering algorithm with optimal parameters for reducing the energy consumption and prolonging the system lifetime. The proposed algorithms only covered energy efficiency at the clustering stage of the sensor nodes.

Ozalp and Tekin (2014) presented a content-aware routing for wireless sensor networks using intelligent agents for efficient data delivery and enhanced network life time. In the proposed network platform, data delivery is performed by the agents but with the decision of the individual nodes. In the proposed model, the network topology is temporal and sensory data are disseminated based on subscription and publishing architecture. This strategy of data communication can result to loss of sensitive data that have over stayed for subscription and publishing. Therefore, it is not a suitable solution for mission critical applications.

Smarsly, Law and König, (2011) showed a resource-efficient wireless monitoring based on mobile agent migration. Their objective was to design a new approach for resource-efficient wireless sensor networks based on a multi-agent paradigm that provides access to restricted computing resources. The reported results in the work only showed reduction in the memory utilization of the networks. Memory is a dependent resource in any wireless network computing environment. Independent (power) resource of the network was not considered in the work. 
Chavhan and Venkataram (2015) proposed a scheme that provides Quality of Service (QoS) routing in mobile ad hoc networks though periodic interactions among agents that are running on the sensor nodes that form the network. Static agents serve as cluster heads in the scheme while mobile agents explore the network for data gathering through interactions with other agents.

Dong et al., (2014) showed a mobile agent-based and efficient data-gathering system in wireless sensor. Their technique makes use of mobile agents for data collection and mobile server for storage of the gathered information. One hop communication as considered in the work is not energy efficient for large scale wireless sensor networks.

\section{The Proposed Solution}

\subsection{Characteristics of WSN}

Typically, wireless sensor networks are identified with the following characteristics.

- Power consumption constrains for nodes using batteries

- Ability to cope with node failures

- Mobility of nodes

- Dynamic network topology

- Communication failures

- Heterogeneity of nodes

- Scalability to large scale of development

- Ease of use

- Unattended operation

Sensor networks are a promising approach for a variety of applications that cut across industrial application, security and surveillance, precision agriculture, health application, smart home, environmental monitoring, structural monitoring, fire detection and many more.

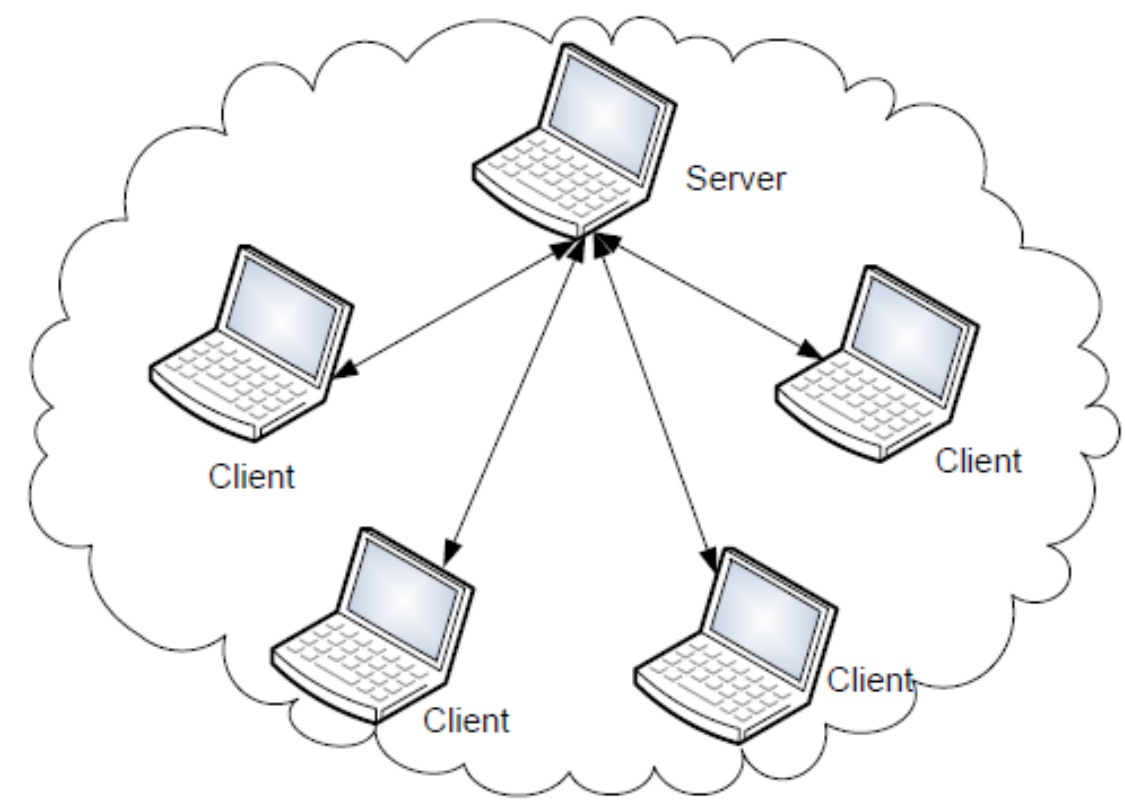

Fig. 1. Server/client based model. Source: Authors. 
Figure 1 shows a general architecture of server/client model. In this model, connection and transmission of data only exists between a client and the server and not between a client and other clients. This means, clients totally depend on the server and vice versa. It also implies that a client (server) needs to wait for a response from the server (client). This mode has execution time and energy consumption limitations if the number of nodes is quite large.

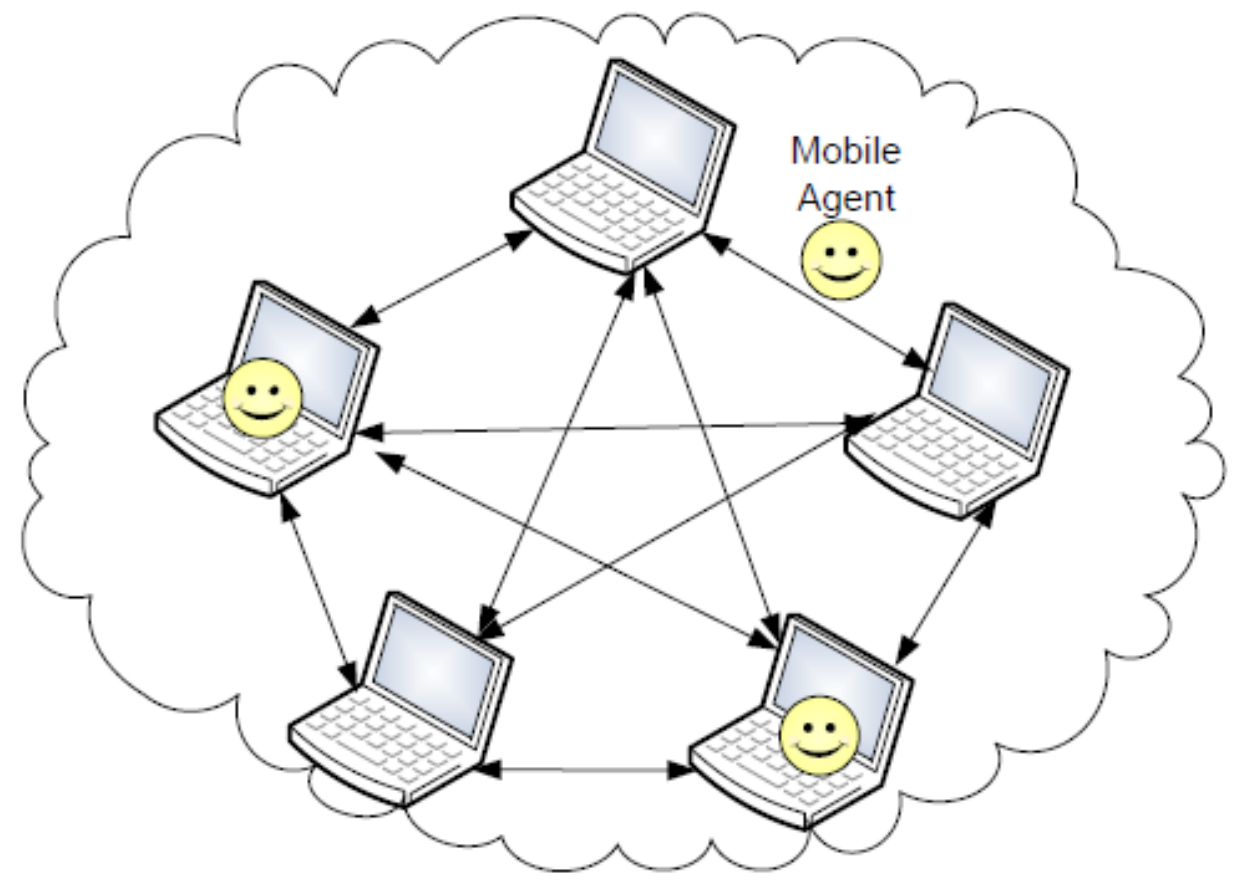

Fig. 2. Mobile agent-based model. Source: Authors.

Figure 2 shows the architecture of mobile agent-based model. Computers are connected to a wireless network in both cases. As shown in Figure 2, each MA can run its process independently on each computer. Therefore, the MA model tends to provide more timeefficient performance of a network than the server/client model.

The current focus in wireless sensor networks is placed on the operations of the network with energy-efficiency, dependability, timeliness and scalability. The availability of various sensors that can be networked using the wireless technology allows for large-scale sensing of physical spaces. The accuracy and the timeliness of sensed information are extremely important in all many applications; since crucial decisions will be taken based on these sensed values. In a multi-hop environment, where the sink reaches the source through multiple hops, nodes closer to the sink nodes drain their power budgets faster.

\subsection{Solution Algorithms}

The node modes of operation are represented by the states of a Markov chain and the random variables represent the probability of staying at each state at a particular time.

Using this model, we will have a sequence of random variables $X_{0}, X_{1}, X_{2}, \ldots X_{n}$ that represents its states during the time.

Then;

$$
X_{n}=i
$$


where $i$ is the mode operation at time-step ${ }^{3} \mathrm{n}$

At each time the node is in state $i$, there is a fixed probability, $\mathrm{P}_{i j}$, that the next state will be $j$. this probability is represented as

$$
\mathrm{P}_{i j}=P\left\{X_{m+1}=j \mid X_{m}=i\right\}
$$

Defining the n-step transition probability, $\mathrm{P}_{i j}{ }^{(\mathrm{n})}$ that a node presently in state $i$ will be in state $j$ after $n$ additional transitions

$$
\mathrm{P}_{i j}^{(\mathrm{n})}=\sum_{k=1}^{M} P_{\mathrm{ik}}{ }^{(\mathrm{r})} P_{\mathrm{kj}}^{(\mathrm{n}-\mathrm{r})} \text {, for any value of } 0<r<n
$$

If a node is in state $i\left(X_{0}=1\right)$, the number of time-steps a node will stay in the state $s$ can be calculated by $\sum_{t=1}^{T} P_{i s}{ }^{(\mathrm{t})}$

If $E_{s}$ is the amount of energy dissipated by a node that remains one time-step in state $s$, and the node is currently in state $i$, then the expected amount of energy spent in the next $T$ times, $E^{T}$

$$
E^{T}=\sum_{s=1}^{M}\left(\sum_{t=1}^{T} P_{\text {is }}^{\left({ }^{(t)}\right.}\right) \times E_{s}
$$

\subsection{Nodes Clustering}

Each node in the sensor field has certain capabilities to process the sensed data. The task at this level is to group the nodes in the sensory field into a number of clusters. Suppose that an intelligent mobile agent must visit a certain number of nodes in order to complete an assigned task. The procedure will be to find cluster centre $C_{r}$ and then cluster all alternative nodes.

We define similarity measurement $d(X, C)$ as the generalized Euclidean distance between nodes $X_{i}$ and $C_{r}$, where $\mathrm{r}=1,2,3, \ldots, n$

$d\left(X_{i}, C_{r}\right)=\sqrt{k_{1}\left(x_{1}-c_{11}\right)^{2}+k_{2}\left(x_{2}-c_{12}\right)^{2}+\cdots+k_{m}\left(x_{m}-c_{1 m}\right)^{2}}$

Where $\mathrm{k} 1, \mathrm{k} 2, \ldots, \mathrm{km}$ are the generalized Euclidean distance, where the coefficients

$$
\mathrm{k}=\left\{k_{i}: i=1, \ldots m\right\}
$$

A node $X$ belongs to cluster $r$ if and only if $d\left(X, C_{r}\right)<d\left(X, C_{t}\right) \mid t=1,2, \ldots, R$ and $t<r$ 


\subsection{Energy Consumption Model}

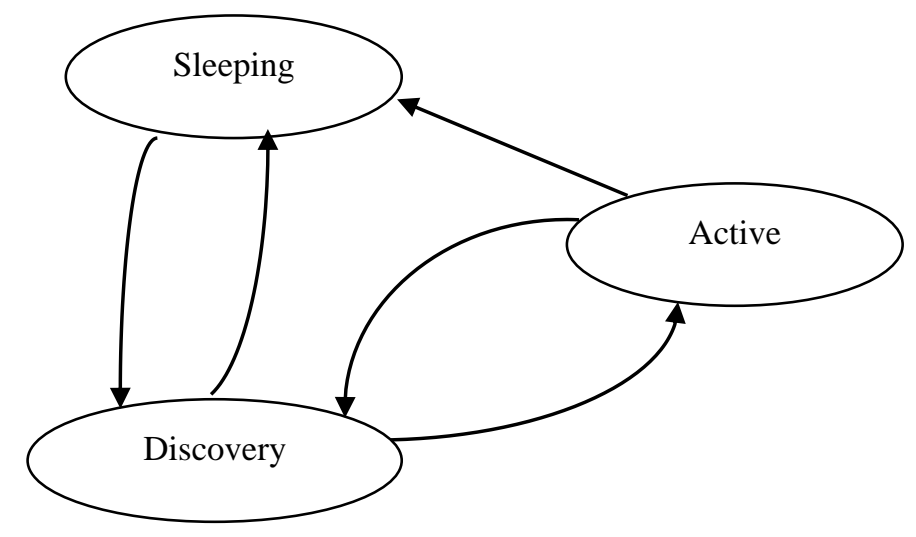

Fig. 3. Sensor mode of transition. Source: Authors.

One duty cycle includes one sleep period of $s$ and one active period of $(T-s)$. The probability $p$ that the wireless node cannot be connected is

$$
P=\frac{s}{T}
$$

The minimum active period $(T-s)$ has to be at least twice that of the transmission time of one mobile agent so that there is enough time for the mobile agent to be transmitted successfully to the next wireless node. If we have congestion probability as $P$ and $(T-s)=2 \Delta$, we have

$$
T=\frac{2 \Delta}{1-P} \text { and } s=\frac{2 P \Delta}{1-P}
$$

To compute the energy needed for a mobile agent traversing a set of nodes; in this case, all wireless nodes have a regular duty cycle with one sleep mode and one active mode. Conventionally, sensor nodes are design to go into sleep state when there are no sensing activities going on in the target region and to resume sensing when there are observable actions. We assume that the state of the source node carrying the mobile agent during this predetermined period of sleeping and waking as the energy consumed during the normal duty cycle. The energy in the normal duty cycle is denoted by Enormal expressed as

$$
\mathrm{E}_{\text {normal }}=\left(\mathrm{s} E_{s}+(T-s) E_{a}=2 \mathrm{n} \Delta\left(\frac{p E_{S}+(1-p) E_{a}}{1-p}\right)\right.
$$

where $E_{s}$ and $E_{a}$ represent the utilized energy at sleep mode and active mode.

Mobile agents transit through the network and not all the sensor nodes that are in the active mode of housing the mobile agents at the same time. Therefore, sensors with mobile agents must come out of sleeping mode for prompt communication of the gathered data to the processing element. 
The node with the mobile agent has to be in the active mode during the whole duty cycle in order to avoid loss of the mobile agent. Hence, the energy in the special duty cycle $\mathrm{E}_{\text {special }}$ is

$\mathrm{E}_{\text {special }}=(\mathrm{n}-1)\left(s P_{s}+(T-s) P_{a}\right)+T P_{a}=2(\mathrm{n}-1) \Delta\left(\frac{p P_{s}+(1-p) P_{a}}{1-p}\right)+2 \Delta \frac{P_{a}}{1-p}$

For a mobile agent to successfully obtain sensors' information, we can have $r_{1}$ normal duty cycles and $r_{2}$ special duty cycles, and the total energy required is

$$
\mathrm{E}_{\text {total }}=\left(\mathrm{r}_{1}+1\right) \mathrm{E}_{\text {normal }}+(\mathrm{r} 2-1) \mathrm{E}_{\text {special }}
$$

The total energy consumption in the cluster model is affected by the transmission distance between the internal node serving the MA and the cluster head. Since any MA visits a cluster head at least two times when the cluster head receives/sends back the MA from/to the initiating node, the total energy consumption in the cluster model is given by:

$$
\text { Ecluster }=\sum_{i=1}^{M}\left\{n_{i} E_{M A}+2 E_{a m p} D_{i}^{\alpha}\right\}
$$

where $D_{i}$ is the transmission distance between the server and a cluster head dispatched by MA $i, \alpha$ is the exponent of the path loss propagation $(2 \leq \alpha \leq 5)$, and Eamp is a constant.

\section{Discussion and conclusion}

Wireless sensor network is a sensitive and high resource constrained network. Conceptually, conventional sensor networks designed without sleeping and active modes of sensors can lead to slightly uniform energy depletion. Therefore, the transition probabilities of sensors can prolong the lifetime of the network if the sensor node currently hosting the mobile agent is not on sleep mode. Cluster leaders are selected based on the signal strength of the nodes and the clustering technique optimizes data transmission. By using sleep/active Algorithm in largescale wireless sensor networks, energy consumption is reduced and network life time is increased. The proposed model shows that we can determine an efficient duty cycle for heterogeneous sensor monitoring.

By moving the processing code to the data, a MA can avoid the transmission of intermediate data in the network, continue working even in the presence of disconnections in the network, and then complete the entire task faster than the Client/Server traditional solutions. Our approach is used for continuous applications where nodes periodically generate data, and also for event-driven applications where nodes generate data when an event arises in the target field. Our approach will extend the network lifetime by using an equitable energy dissemination approach which limits the energy cost and communication while maintaining a balance of energy consumption between all the participating nodes. The proposed solution will perform multiple tasks associated with small amounts of data carried by a single agent. The result will be concatenated into a single package to give a better aggregation in order to reduce the communication cost. 
As the energy factor is one of the most important constraints that guide the design of protocols in wireless sensor networks, every attempt to design these networks must incorporate mechanisms that will allow users to extend the lifetime of the entire network. We have proposed an optimal strategy for organizing sensors into a hierarchy of clusters with an objective of minimizing the total energy spent in the system to communicate the information gathered by these sensors to the processing element. In the subsequent research work, we intend to consider an underlying medium access protocol and investigate how that would affect the optimal probabilities of a node becoming a cluster head and the run-time of the algorithm.

\section{References}

Ahmed, A. A. (2013). An enhanced real-time routing protocol with load distribution for mobile wireless sensor networks. Computer Networks, 57(6), 1459-1473. doi: 10.1016/i.comnet.2013.02.003

Alipour, M. \& Faez, K. (2011). On Design of Mobile Agent Routing Algorithm for Information Gain Maximization in Wireless Sensor Networks. In The Sixth International Conference on Systems and Networks Communications, (pp. 193-198). IARIA. Retrieved from https://www.thinkmind.org/download.php?articleid=icsnc 20118

Chavhan S. \& Venkataram P. (2015). Emergent Intelligence Based QoS Routing in MANET. Procedia Computer Science, 52, 659-664. doi: 10.1016/j.procs.2015.05.068

Dong, M, Ota, K., Yang, L. T., Chang, S., Zhu, H. \& Zhou, Z. (2014). Mobile Agent-Based EnergyAware and User-Centric Data Collection in Wireless Sensor Networks. Journal of Computer Networks, 74(Part B), 58-70. doi: 10.1016/j.comnet.2014.06.019

Imianvan, A. A. (2008). Development of a mobile agent for evaluating the use of bandwidth in a computer network. PhD Thesis. Department of Computer Science, Federal University of Technology, Akure, Nigeria.

Lange, D. B. \& Oshima, M. (1999). Seven Good Reasons for Mobile Agents. Communications of the ACM, 42(3), 88-89. doi: $10.1145 / 295685.298136$

Lin, K., Chen, M., Zeadally, S. \& Joel, J. P. C. (2012). Balancing energy consumption with mobile agents in wireless sensor networks. Future Generation Computer Systems, 28(2), 446-456. doi: 10.1016/j.future.2011.03.001

Mini, R. A. F., Machado, M. do V., Loureiro, A. A. F. \& Badri N. (2003). Prediction-based Energy Map for Wireless Sensor Networks. Ad Hoc Networks, 3(2), 235-253. doi: 10.1016/j.adhoc.2004.07.008

Chen, M., Gonzalez, S. \& Leung, V. C. M. (2009). Applications and Design Issues for Mobile Agents in Wireless Sensor Networks. IEEE Wireless Communications, 14(6). 20-26. doi: 10.1109/MWC.2007.4407223

Ozalp, N. \& Tekin Y. (2014). Content-Based Routing for Wireless Sensor Networks using Intelligent Agents. In IEEE 15th International Symposium on Computational Intelligence and Informatics (pp. 345-350), New York: IEEE. doi: 10.1109/CINTI.2014.7028700

Smarsly, K., Law, K. H. \& König, M. (2011). Resource-Efficient Wireless Monitoring based on Mobile Agent Migration. Structural-health-monitoring.com. Retrieved from http://eil.stanford.edu/publications/kay smarsly/smarsly2011c.pdf

Sutagundar, A.V. \& Manvi, S.S. (2013). Location aware event driven multipath routing in Wireless Sensor Networks: Agent based approach. Egyptian Informatics Journal, 14(1), 55-65. doi: $\underline{\text { 10.1016/i.eii.2013.01.003 }}$

Zhu, C., Zhenga, C., Shuc, L. \& Han, G.. (2012). A survey on coverage and connectivity issues in wireless sensor networks. Journal of Network and Computer Applications, 35(2), 619-632. doi: 10.1016/j.jnca.2011.11.016 
